\title{
Efeito de jogo de futsal sobre o desempenho intermitente de alta intensidade em futebolistas amadoras
}

Futsal game effect on high-intensity intermittent performance in amateur female soccer players

\author{
Izabela Aparecida dos Santos ${ }^{1, *}$ \\ Marina de Paiva Lemos ${ }^{1}$ \\ Gustavo Ribeiro da Mota ${ }^{1}$
}

\section{Resumo}

Objetivo: Avaliar o efeito agudo de um jogo simulado de futsal sobre o desempenho intermitente de alta intensidadee indicadores fisiológicos em futebolistas amadoras. Métodos: Dezesseis futebolistas (22,5 $\pm 1,7$ anos)realizaram o teste YoYolntermitenteEndurance 2 (YoYolE2) em duas situações distintas (em dias separados por 48-h): baseline (descansadas e recuperadas) e logo após jogo amistoso de futsal. Resultados: A distância percorrida no YoYolE2 na baseline foi de $118,8 \pm 4,6 \mathrm{~m}$ e, logo depois do jogo, foi de71,2 $\pm 5,4 \mathrm{~m}$ (queda de $\sim 40 \% ; \mathrm{p}<0,05$, tamanho do efeito = 9,5). Logo após o jogo, indicadores de intensidade relativa aumentaram $(p<0,05)$ (Frequência cardíaca [FC] média/recuperação e percepção de esforço) em comparação com o YoYolE2 basal - exceto FCpico.Conclusão: O desempenho intermitente de alta intensidade (YoYolE2) é fortemente reduzido por uma partida simulada de futsal e variáveis associadas a intensidade interna bem como a recuperação aguda são prejudicadas pelo jogo. Portanto, YoYolE2 é sensível para detectar alterações físicas agudas em futebolistas amadoras.

Palavras-chave: desempenho, fadiga, futsal.

Afiliação dos autores

${ }^{1}$ Programa de Pós-Graduação em, Educação Física, Universidade Federal do Triângulo Mineiro, Uberaba, Minas Gerais, Brasil.

${ }^{*}$ Autor correspondente

Programa de Pós-Graduação em Educação Física Universidade Federal do Triângulo Mineiro, Av. Tutunas, 490, CEP: 38061-500, Uberaba, Minas Gerais, Brasil. e-mail:

\section{Abstract}

Objective: To evaluate the acute effect of a simulated futsal game on high-intensity intermittent performance in amateur soccer players. Methods: Sixteen female soccer players $(22.5 \pm 1.7$ years) performed the YoYo Intermittent Test Endurance Test 2 (YoYolE2) on two distinct days (48-h apart): baseline (rested and recovered) and just after a friendly futsal game. Results: The distance covered in YoYolE2 in the baseline was of $118.8 \pm 4.6 \mathrm{~m}$ and just after the game $71.2+5.4 \mathrm{~m}(\sim 40 \%$ drop; $p<0.05$, effect size $=9.5)$. Just after the game, relative intensity indicators increased $(p<0.05)$ (Heart rate [HR] mean/recovery and perceived exertion) in comparison with baseline YoYolE2- except HRpeak. Conclusion: Intermittent high-intensity performance (YoYolE2) is strongly reduced by a simulated game of futsal and variables associated with internal intensity as well as acute recovery are impaired by the game. Therefore, YoYolE2 is responsive to detect acute physical changes in amateur soccer players.

Keywords: performance, fatigue, futsal.

Introdução izabelaeduca94@hotmail.com.

Os autores declararam não haver conflito de interesses.

Processo de arbitragem

Recebido: 03/01/2018 Aprovado: 08/02/2018
Conflito de interesses 
O futebol e futsal são modalidades intermites de alta intensidade e se assemelham de maneira física e fisiológica, além da proximidade motora ${ }^{1}$. Apesar da popularização do futsal, há escassez de estudos para melhor compreensão, monitoração e elaboração de estratégias de treinamentoacerca dessa modalidade.

Desempenhar corridas intermitentes de alta intensidade é exigência que deve ser desenvolvida por futebolistas em diferentes níveis. Inclusive, um dos parâmetros para distinguir a condição física de atletas é a análise do desempenho em distâncias totais cobertas ${ }^{2}$

Uma partida de futebol feminino indica alta exigência física para maioria das futebolistas envolvidas, embora essa exigência possa variar de acordo com a posição no jogo, bem como pelo status de treinamento do indivíduo ${ }^{3}$. Resultado dessa elevada demanda física é a queda de desempenho que ocorre nas etapas finais de um jogo, claramente observadas em jogos de elite no futebol masculino e feminino ${ }^{4,5,6}$ e também em indicadores de fadiga associados ao jogo de futebol para populações especiais como, por exemplo, futebol para amputados ${ }^{7,8,9}$. $^{2}$

Estudos discutem esse acontecimento como resultado de fadiga fisiológica, elementos táticospsicológicos e de contexto real dos jogos incluindo, por exemplo, maior ou menor posse de bola ${ }^{10}$. Além disso, sabe-se que o desempenho em corridas de alta intensidade durante jogos está diretamente ligado ao nível de treinamento. Por exemplo, futebolistas que possuem alto padrão de competitividade, executam significativamente maiscorridas de alta intensidade quando comparados a de níveis inferiores ${ }^{11,12}$. Porém, ao investigarmos o futsal essas comparações são inexistentes.

O teste denominado YoYo intermitente endurance nível 2 (YoYolE2) é altamente recomendado para futebolistas, pois avalia o estado de treinamento específico em diferentes níveis para ambos os sexos e é altamente reprodutível e associado ao desempenho em jogos. Ou seja, quanto maior a distância percorrida no YoYolE2, maior o número de corridas em alta intensidade realizadas pelos futebolistas em jogos ${ }^{13,14}$.

Apesar da inegável utilidade do YoYolE2 na rotina de futebolistas por ser simples, reprodutível e específico ${ }^{15}$, não sabemos qual a sensibilidade deste teste para quantificar fadiga específica. Ou mesmo se ele (YoYolE2) é capaz de detectar pioras no rendimento de maneira aguda. Assim, o objetivo do presente estudo foi avaliar o efeito agudo de um jogo simulado de futsal sobre o desempenho intermitente de alta intensidade (YoYolE2) em futebolistas amadoras. Nossa hipótese foi que o jogo causaria redução aguda no desempenho do YoYolE2 por gerar fadiga e, portanto, o teste seria considerado sensível para quantificar tais reduções.

\section{Métodos}

\section{Amostra e cuidados éticos}

Participaram do estudo dezesseis jogadoras amadoras de futsal com idadede $22,5 \pm 1,7$ anos $(1,61 \pm 0,52 \mathrm{~m} ; 63,8 \pm 3,32$ $\mathrm{kg}$;́ndice de massa corporal $=23,9 \pm 2,01 \mathrm{~kg} / \mathrm{m}^{2}$ ). As jogadoras treinavam três vezes semanais (seis horas/semana) e não possuíam lesões musculoesqueléticas ou alguma outra limitação que prejudicasse a realização do teste. Pelas demandas físicas e fisiológicas, os dados das goleiras foram excluídos deste trabalho. O estudo foi aprovado pelo comitê de ética e pesquisa da Universidade Federal do Triângulo Mineiro, sob o parecer de $n^{\circ} 993.636 / 2015$. Antes do início da pesquisa, todas voluntárias foram informadas sobre os objetivos e procedimentos do estudo. Após o aceite verbal de participação, assinaram o Termo de Consentimento Livre e Esclarecido.

\section{Desenho experimental}

Após o processo de familiarização, na primeira sessão (baseline), ao chegarem, as futebolistas respondiam individualmente (para evitar influência de outras jogadoras) a escala de percepção subjetiva de recuperação (PSREC). Em seguida, iniciavam o aquecimento e o teste YoYolE2. Durante todo o aquecimento, teste e logo após o mesmo (recuperação aguda) a frequência cardíaca (FC) foi registrada. Passados vinte minutos, individualmente foi registrada a percepção subjetiva de esforço (PSE) referente ao teste. Após a primeira sessão, as futebolistas foram instruídas a absterem do uso de bebidas energéticas, alcoólicas, cafeinadas, bem como, manter alimentação habitual e evitar exercícios vigorosos no período de 48 horas conforme recomendam estudos envolvendo mensurações fisiológicas e de desempenho ${ }^{16,17,18}$.

Após 48-h iniciou-se a segunda sessão. A PSREC foi registrada novamente seguindo o mesmo padrão citado anteriormente. Logo após as futebolistas foram separadas em dois times, no qual realizaram jogo de futsal amistoso. Durante o jogo a FC foi registrada também. Dez minutos após o encerramento da partida, as futebolistas realizaram o teste YoYolE2. Completado 20 minutos após teste, todas responderam individualmente a escala PSE.

\section{Familiarização}

Antes de iniciar a coleta de dados, todas as futebolistas $(n=16)$ realizaram três sessões de familiarização (em dias distintos) para conhecerem as escalas de percepções, bem como o teste (YoYolE2). Tal procedimento é necessário para garantir o aprendizado e evitar diferenças nas mensurações por efeito de aprendizagem ${ }^{19,20}$.

\section{Percepção subjetiva de recuperação (PSREC)}

A escala de percepção subjetiva de recuperação (PSREC) (21)que apresenta numeração de 6 a 20 (6 significa "em nada recuperado" e 20 "totalmente bem recuperado") foi aplicada antes de quaisquer atividades (em todas as sessões)como forma de registrar o status de recuperação percebido pelas futebolistas.

\section{YoYo intermitente endurance nível 2 (YoYolE2)}

Apenas para o baseline foi realizado o aquecimento do YoYolE2, que consistiu na realização dos três primeiros níveis do teste. O YoYolE2 consiste em correr repetidamente (em linha reta) $2 \times 20$ metros $(\mathrm{m})$ em estágios de velocidade progressivamente crescentes (velocidade inicial $12 \mathrm{~km} . \mathrm{h}-1$ ) corrida de frente, guiados por áudio específico (5 segundos para recuperação em uma área marcada de $2,5 \times 2 \mathrm{~m}$ atrás da linha de $20 \mathrm{~m}$ demarcada). A interrupção (e final) do teste se deu pela falta de alcance da metragem e/ou do ritmo duas vezes, registrando o último estágio completo que o indivíduo percorreu $^{22}$. Com 0 número de estágios realizados, calculamos a distância total percorrida para cada futebolista. Com o propósito de evitar quaisquer efeitos placebo ou nocebo, comuns em estudos com testes de desempenho físico $^{23,24}$ as jogadoras não foram informadas sobre 0 real objetivo do presente trabalho. Apenas foi ressaltado que o objetivo era realizar o melhor desempenho possível nos testes. Também houve o "cegamento" das participantes em relação ao desempenho no teste (ou seja, não foi revelado qual o estágio alcançado nos testes e valores de FC) para evitar potencial "dosagem" de ritmo.

\section{Jogo de futsal}

Após responderem a PSREC, as futebolistas foram separadas pela técnica responsável de maneira equilibrada (aspectos físico, tático e técnico) em dois times. Esses times realizaram jogo de futsal amistoso em quadra poliesportiva medindo 42 por 21 metros, com piso de concreto cimentado e pintado, às 19 horas (horário de Brasília), temperatura em $23^{\circ} \mathrm{C}$ e umidade relativa do ar de $62 \%$. A partida foi realizada em 2 tempos de 20 minutos com intervalo de 10 minutos seguindo todas regras oficiais da confederação brasileira de futsal (CBFS), exceto as substituições que não foram permitidas.

Frequência cardíaca (FC) e percepção subjetiva de esforço (PSE) 
A FC foi registrada ao longo do teste e após (recuperação aguda- 1 e 3 minutos), utilizando o monitor cardíaco Polar Team System ${ }^{\circledR}$, composto por fitas na altura do tórax da participante. Após YoYolE2, a futebolista indicava (individualmente para evitar a influência de outras) um valor para seu esforço percebido por meio da escala CR-10 Borg para determinar a intensidade subjetiva e interna da sessão. Esta escala varia de 0 a 10 , onde 0 é "nada" e 10 é "muito muito difícil (máximo)"

\section{Análise estatística}

Foi aplicado o teste de Shapiro-Wilk para verificar a normalidade dos dados. $O$ teste $T$ pareado (dados paramétricos) foi aplicado para comparar os resultados dos dois momentos baseline versus após o jogo. Os dados são apresentados em média \pm desvio padrão. O tamanho do efeito, ou effect size (ES), foi calculado de acordo com Cohen (1988) para determinar a significância das diferenças encontradas no teste de desempenho físico e classificadas como: trivial $(<0,2)$, pequeno $(>0,2-0,6)$, moderado $(>0,6-1,2)$, grande $(>1,2-2,0)$ e muito grande $(>2,0)$ com base em recomendações específicas para a Ciência do Esporte (26). O nível de significância adotado foi de $5 \%$ e utilizamos 0 software GraphPad ${ }^{\circledR}$ (Prism 6.0, San Diego, CA,USA).

\section{Resultados}

A PSREC não diferiu $(P>0,05)$ entre baseline vs dia do jogo(14,5 \pm 0,3 vs 14,1 $\pm 0,3 \cup A)$.

A FC média da partida foi de $175,9 \pm 4,1$ ( $89 \%$ da FCmax) e a FCpico (a mais alta registrada)foi de 183,2 $\pm 4,5$ (92\% da FCmax).

A Figura 1 ilustra a distância total percorrida no YoYolE2 nos dois momentos (descansadas, em dia anterior e logo após o jogo). $O$ teste $T$ pareado mostrou reduçãosignificativana distância percorrida $(\mathrm{P}<0,05)$, passando de $118,8 \pm 4,6 \mathrm{~m}$ baseline para $71,2 \pm 5,4 \mathrm{~m}$ após o jogo ( $-40 \%)$. Ot amanho do efeito encontrado foi de 9,5 (muito grande).

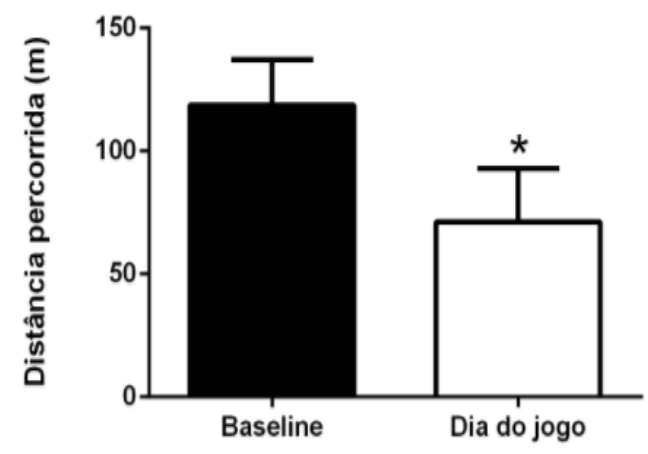

Figura 1. Distância total percorrida no teste YoYo endurance nível 2 (metros) em estado basal (baseline) e depois de um jogo de futsal. ${ }^{*}=p<0,05$ em comparação ao primeiro momento.

A Tabela 1apresenta as respostas de FC e PSE ao YoYolE2, tamanho do efeito e classificação das mesmas. Não foi encontrada diferença na FC pico, já nas demais foram encontradas comparando as duas ocasiões $(p<0,05)$.

Tabela 1

Respostas da FC e PSE ao teste YoYolE2.

\begin{tabular}{lcc} 
& & \\
\hline FC pico YoYolE2 (bpm) & $184,6 \pm 1,0$ & Após o jogo \\
FC média YoYolE2 (bpm) & $172,8 \pm 1,5$ & $186,6 \pm 1,1$ \\
FC pós 60 s YoYolE2 (bpm) & $125,7 \pm 1,0$ & $158,8 \pm 0,7^{*}$ \\
FC pós 180 s YoYolE2 (bpm) & $94,4 \pm 0,8$ & $108,3 \pm 1,2^{*}$ \\
PSE (UA) & $8,8 \pm 0,2$ & $9,6 \pm 0,1^{*}$ \\
\hline
\end{tabular}

${ }^{*} \mathrm{p}<0,05$.

\section{Discussão}

O objetivo do estudo foi avaliar 0 desempenho intermitente de alta intensidade em futebolistas amadoras após jogo de futsal simulado. Nosso principal achado foi que o jogo causou queda na performancedo teste (- $40 \%$ no YoYolE2) e elevou indicadores de intensidade interna (FC média, FC de recuperação e PSE). Tais resultados são fruto da fadiga induzida pelo jogo.

Como a FC durante o jogofoi relativamente elevada (FC média $=175,9 \pm 4,1-89 \%$ da FCmáx e pico=183,2 $\pm 4,5-92 \%$ da FCmáx), podemos afirmar que apesar de se tratar de um jogo simulado foi uma partida de alta intensidadepara todas participantes. Adicionalmente, por não serem permitidas substituições a FC média foi ligeiramente superior àquela normalmente encontrada em jogos de futsal com possibilidade de substituição ${ }^{27}$, a zona de FC ficou em aproximadamente $90 \%$ da FC máxima (calculado pela formula 220 -idade das participantes).

Em um estudo ${ }^{18}$ corroborando com nossos achados, foi observada queda $60 \%$ no desempenho do YoYolE2 após partida competitiva de futebol feminino de elite. No presente estudo a diminuição em termos percentuais foi de $40 \%$. 0 menor valor percentual aqui reportado provavelmente se deve ao maior dano muscular causado pelo futebol em comparação às outras modalidades esportivas coletivas ${ }^{28}$. O maior dano muscular gerado pelo futebol faz sentido porque está relacionado ao volume e trabalho mecânico da musculatura ${ }^{29}$. No futebol é comum maior volume, com distâncias totais percorridas acima de $10 \mathrm{~km}$ (por jogo) ${ }^{10,30}$ em comparação ao futsal 3,5 a $4 \mathrm{~km}$ (por jogo) ${ }^{30}$. Em relação à distância percorrida no YoYolE2 do atual trabalho, notamos grande discrepância com a pesquisa de Krustup (2010), mas isso se explica pelo fato que atletas de futebol feminino têm melhor desempenho do teste quando comparado as de futsal ${ }^{31}$. Ademais, a nossa amostra é composta por futebolistas amadoras e não profissionais como no estudo de Krustup (2010) o que comprovaque o status de treinamento influencia o desempenho do teste. Essa comum queda no desempenho seja no futebol ou no futsal como encontramos evidencia que o tipo de fadiga que ocorre nos últimos estágios do jogo e após tem repercussão na capacidade de recuperação entre exercícios intermitentes exigidos na partida.

A FC assim como PSE (criada a partir da FC), são métodos para quantificar a intensidade interna do exercício ${ }^{25}$. No presente estudo a FC pico, ou seja, o maior valor atingido pela FC no momento do YoYolE2 não diferiu nos dois momentos (baseline VS dia do jogo). Em ambas situações os valores foram de $90 \%$ da FC máxima. Isso indica que independente do momento, em termos de intensidade interna o teste é altamente intenso, revelando dados relevantes sobre o teste, bem como sobre o momento para sua aplicação.

Em relação às variáveis de recuperação aguda, nossos dados mostraram que as FC'stanto após 60s quanto após 180 $\mathrm{s}$ foram superiores no teste no dia do jogo do que na situação basal. Provavelmente esses resultados devem se a exaustão imposta pela partida, acrescentado pela maior concentração e processo mais demorado da retirada de metabólitos resultados da maior contração muscular pelo maior trabalho (jogo+teste) e das catecolaminas circulantes. Adicionalmente, fatores como a elevada temperatura corporal acarreta provável estado de desidratação, desencadeando aumento mais acentuado da FC pela redução do volume sanguíneo ${ }^{32,33}$. Vale ressaltar que para jogadores de futsal é essencial a melhora na reativação vagal, pois essa condição representa decréscimo do stress-cardíaco pós-exercício, além de refletir melhor adaptação ao treinamento ${ }^{34}$. Lembramos, entretanto, que este estudo avaliou apenas o momento agudo, mas sugere-se ser o início de uma reativação vagal plena.

A PSE foi aplicada após 20 minutos do final do teste YoYolE2 e foi apurado uma diferença significativa (dia do jogo maior que baseline) indo de encontro com os achados dos valores de FC's, uma vez que a escala apresenta forte correlação com outros parâmetros internos de intensidade de exercício, por exemplo, o VO2 e $\mathrm{FC}^{35}$. Ou seja, além de fadiga física (desempenho) e fisiológica (FC's), o jogo causa no desempenho intermitente incremento na percepção da fadiga.

Temos que deixar o leitor ciente de algumas limitações do presente estudo. Uma é a ausência de controle sobre ações 
físicas do jogo: distância total percorrida, número de sprints, velocidade de deslocamento, acelerações, desacelerações, etc. Apesar de termos os valores médio e pico de FC, a quantificação do trabalho físico realizado seria importante para analisar mais profundamente a resposta individual de cada futebolista frente ao teste ao jogo e, logicamente, ao teste. Outra limitação é o fato do jogo realizado ser diferente do jogo real (onde substituições são permitidas). Contudo, frente à escassez de literatura sobre efeito do jogo de futsal em comparação ao futebol, que apresenta estudos em diferentes áreas ${ }^{36,37^{\dagger}}$ e a sensibilidade de teste altamente específico para futebolistas, acreditamos que nosso trabalho acrescenta algo e colabora com a aplicação prática de equipes coletivas.

\section{Conclusão}

O desempenho intermitente de alta intensidade, avaliado pelo YoYolE2, é fortemente reduzido por uma partida simulada de futsal em futebolistas amadoras e variáveis associadas a intensidade interna bem como a recuperação aguda são prejudicadas pela realização do jogo. Assim, concluímos que o YoYolE2 é sensível para detectar alterações físicas agudas em futebolistas amadoras.

\section{Referências}

1. Ré AN. Características do futebol e do futsal: implicações para o treinamento de adolescentes e adultos jovens. Rev Dig EF Desp2008;(13)127.

2. Mohr M, Krustrup P, Andersson H, Kirkendal D, Bangsbo J. Match activities of elite women soccer players at different performance levels. $J$ Strength Cond Res 2008; 1;22(2):341-9.

3. Todd DS, Chisnall P. Fitness characteristics of english female soccer players: an analysis by position and playing standard. Science and football IV 2013; 19:374.

4. Andersson H, Ekblom B, Krustrup P. Elite football on artificial turf versus natural grass: movement patterns, technical standards, and player impressions. J Sports Sci 2008; 26(2):113-22.

5. Mohr M, Krustrup P, Bangsbo J. Match performance of high-standard soccer players with special reference to development of fatigue. J Sports Sci 2003; 21(7):519-28.

6. Krustrup P, Mohr M, Ellingsgaard HE, Bangsbo J. Physical demands during an elite female soccer game: importance of training status. MedSci Sports Exer 2005; 37(7):1242-8.

7. Simim MA, da Mota GR, Marocolo M, da Silva BV, de Mello MT, Bradley PS. The Demands of Amputee Soccer Impair Muscular Endurance and Power Indices But Not Match Physical Performance. AdapPhysActiv Q 2018; 35(1):76-92

8. Simim MA, Bradley PS, da Silva BV, Mendes EL, de Mello MT, Marocolo M, da Mota GR. The quantification of game-induced muscle fatigue in amputee soccer players. J of Sports Med Phys Fitness 2017;57(6):766-72

9. Simim MA, Silva BV, Marocolo Júnior M, Mendes EL, Mello MT, Mota GR. Anthropometric profile and physical performance characteristic of the Brazilian amputee football (soccer) team. Motriz Rev Educ Fís2013;19(3):641-8

10. da Mota GR, Thiengo CR, Gimenes SV, Bradley PS. The effects of ball possession status on physical and technical indicators during the 2014 FIFA World Cup Finals. JSports Sci 2016; 34(6):493-500.

11. Ekblom B. Applied physiology of soccer. Sports Med 1986; 3(1):50-60.

12. Bangsbo J, Nørregaard L, Thorsoe F. Activity profile of competition soccer. Can J Sport Sci 1991;16(2):110-6.

13. Bradley PS, Mohr M, Bendiksen M, Randers MB, Flindt M, Barnes C, et al. Sub-maximal and maximal Yo-Yo intermittent endurance test level 2: heart rate response, reproducibility and application to elite soccer. Eur $\mathrm{J}$ ApplPhysiol 2011; 111(6):969-978.

14. Krustrup P, Bradley PS, Christensen JF, Castagna C, Jackman S, Connolly L, et al. The Yo-Yo IE2 test: physiological response for untrained men versus trained soccer players. Med Sci Sports Exer 2015; 47(1):100-8

15. Marocolo IC, da Mota GR, Londe AM, Patterson SD, Neto OB, Marocolo $M$. Acute ischemic preconditioning does not influence high-intensity intermittent exercise performance. PeerJ2017; 5:e4118 intermittent exercise perform
https://doi.org/ $10.7717 /$ peerj. 4118 .

16. da Mota GR, Magalhães CG, de Azevedo PM, Ide BN, Lopes CR, Castardeli E, Baldissera VV. Lactate threshold in taekwondo through specifics tests. J ExercPhysiology Online 2011; 14(3):60-6

17. da Silva BV, de Moura Simim MA, Marocolo M, Franchini E, da Mota GR. Optimal load for the peak power and maximal strength of the upper body in Brazilian Jiu-Jitsu athletes. J Strength Cond Res2015; 29(6):1616-21.

18. Garcia CA, da Mota GR, Marocolo M. Cold water immersion is acutely detrimental but increases performance post- $12 \mathrm{~h}$ in rugby players. Int $\mathrm{J}$ Sports Med 2016; 37(8):619-24.

19. Meneghel AJ, Verlengia R, Crisp AH, Aoki MS, Nosaka K, Da Mota GR, Lopes CR. Muscle damage of resistance-trained men after two bouts of eccentric bench press exercise. J StrengthCond Res 2014; 28(10):29616.

20. Corrêa da Silva BV, Neme Ide B, de Moura Simim MA, Marocolo M, da Mota GR. Neuromuscular responses to simulated Brazilian jiu-jitsu fights. J Human Kinetics 2014; 44(1):249-57.

21. Kenttä G, Hassmén P. Overtraining and recovery. Sports Med 1998 26(1):1-6

22. Marocolo M, da Mota GR, Pelegrini V, Coriolano HJ. Are the beneficia effects of ischemic preconditioning on performance partly a placebo effect?.Int J Sports Med 2015; 36(10):822-5.

23. Marocolo M, Willardson JM, Marocolo IC, da Mota GR, Simão R, Maio AS. Ischemic preconditioning and placebo intervention improves resistance exercise performance. J Strength Cond Res2016 30(5):1462-9.

24. Marocolo M, Marocolo IC da Mota GR, Simão R, Maior AS, Coriolano $\mathrm{HJ}$. Beneficial effects of ischemic preconditioning in resistance exercise fade over time. Int J Sports Med 2016; 37(10):819-24.

25. Borg E, Kaijser L. A comparison between three rating scales for perceived exertion and two different work tests. Scand J Med Sci Sports 2006;16(1):57-69

26. Batterham AM, Hopkins WG. Making meaningful inferences about magnitudes. Int J Sports PhysiolPerform 2006;1(1):50-7.

27. Miles A, MacLaren D, Reilly T, Yamanaka K. An analysis of physiological strain in four-a-side women's soccer. Science and Football II. London: E \& FN Spon 1993:140-5.

28. Souglis A, Bogdanis GC, Giannopoulou I, Papadopoulos $\mathrm{CH}$, Apostolidis $\mathrm{N}$. Comparison of inflammatory responses and muscle damage indices following a soccer, basketball, volleyball and handball game at an elite competitivelevel. Res Sports Med 2015; 23(1):59-72.

29. Meneghel AJ, Verlengia R, Crisp AH, Aoki MS, Nosaka K, da Mota GR, Lopes CR. Muscledamage of resistance-trainedmenaftertwobouts of eccentricbenchpress exercise. J StrengthCond Res2014; 28(10):2961-6.

30. Stølen T, Chamari K, Castagna C, Wisløff U. Physiology of soccer. Sports Med 2005; 35(6):501-36

31. Clemente FM, Nikolaidis PT. Profile of 1-month training load in male and female football and futsal players. SpringerPlus 2016; 5(1):694.

32. Oliveira RS, Vitor-Costa M, Nakamura FY, Pedro RE, Milanez VF, Bortolotti $H$. Relação entre variabilidade da frequência cardíaca e aumento no desempenho físico em jogadores de futebol. Rev. Bras. Cineantropom. Desempenho Hum 2012; 14(6):713-22.

33. Coyle EF, Montain SJ. Thermal and cardiovascular responses tofluidreplacementduring exercise. Exercise, Heat, and Thermoregulation. Dubuque, IA: Brown and Benchmark 1993;179-212.

34. Goldberger JJ, Le FK, Lahiri M, Kannankeril PJ, Ng J, Kadish AH. Assessment of parasympatheticreactivationafter exercise. American $\mathrm{J}$ of Phys-Heart and CircPhys 2006; 290(6):446-52.

35. Herman L, Foster C, Maher MA, Mikat RP, Porcari JP. Validity and reliability of the session RPE method for monitoring exercise training intensity. South Afri J Sports Med 2006; 18(1):14-7.

36. da Mota GR, Gomes LH, Castardeli E, Bertoncello D, José E, Vicente D, Junior MM, Orsatti FL. Treinamento proprioceptivo e de força resistente previnem lesões no futebol. J Health Scilnst 2010; 28(2):191-193.

37. Germano MD, de Mattos RS, Sindorf MA, Marchetti PH, Verlengia R, Lopes CR, et al. Effects of pre-season shorttermdailyundulatingperiodized training on musclestrength and sprin performance of under-20 soccer players. InternationalJournal of Science CultureandSport (IntJSCS) IntJSCS 2015;3(2):64-72. 